\title{
Classification and reporting guidelines for the pathology diagnosis of placenta accreta spectrum (PAS) disorders; recommendations from an expert panel
}

Running Title: PAS consensus panel consensus document.

Authors:

\begin{tabular}{|c|c|c|}
\hline Author & e-mail & Affiliation \\
\hline $\begin{array}{l}\text { Jonathan L Hecht MD } \\
\mathrm{PhD} \text { (corresponding } \\
\text { author) }\end{array}$ & jlhecht@bidmc.harvard.edu & $\begin{array}{l}\text { Beth Israel Deaconess Medical } \\
\text { Center, Harvard University, Boston } \\
\text { MA (Pathology) }\end{array}$ \\
\hline Rebecca Baergen MD & rbaergen@,med.cornell.edu & $\begin{array}{l}\text { Weill Cornell Medicine, New York } \\
\text { Presbyterian Hospital, New York, } \\
\text { NY (Pathology) }\end{array}$ \\
\hline Linda M Ernst MD MHS & lernst@northshore.org & $\begin{array}{l}\text { NorthShore University } \\
\text { HealthSystem, Evanston, IL and } \\
\text { University of Chicago Pritzker } \\
\text { School of Medicine, Chicago, IL } \\
\text { (Pathology) }\end{array}$ \\
\hline Philip J. Katzman MD & $\begin{array}{l}\text { Philip_Katzman@URMC.roc } \\
\text { hester.edu }\end{array}$ & $\begin{array}{l}\text { University of Rochester Medical } \\
\text { Center, Rochester NY (Pathology } \\
\text { \& Laboratory Medicine) }\end{array}$ \\
\hline Suzanne M Jacques, MD & sjacques@med.wayne.edu & $\begin{array}{l}\text { Hutzel Women's Hospital, Wayne } \\
\text { State University, Detroit MI } \\
\text { (Pathology) }\end{array}$ \\
\hline $\begin{array}{l}\text { Eric Jauniaux, MD, PhD, } \\
\text { FRCOG }\end{array}$ & e.jauniaux@ucl.ac.uk & $\begin{array}{l}\text { University College London, UK } \\
\text { (EGA Institute for Women's } \\
\text { Health, Faculty of Population } \\
\text { Health Sciences) }\end{array}$ \\
\hline $\begin{array}{l}\text { T. Yee Khong MBChB, } \\
\text { MSc, MD, FRCPath }\end{array}$ & $\begin{array}{l}\text { yee.khong@adelaide.edu.au } \\
\text { ORCID ID: 0000-0002-2404- } \\
\text { 007X }\end{array}$ & $\begin{array}{l}\text { Women's and Children's Hospital, } \\
\text { North Adelaide, Australia } \\
\text { (Pathology) }\end{array}$ \\
\hline Leon A. Metlay MD & $\begin{array}{l}\text { Leon_Metlay@urmc.rocheste } \\
\underline{\text { r.edu }}\end{array}$ & $\begin{array}{l}\text { University of Rochester Medical } \\
\text { Center, Rochester NY (Pathology) }\end{array}$ \\
\hline Liina Poder MD & Liina.Poder@ucsf.edu & $\begin{array}{l}\text { University of California San } \\
\text { Francisco, San Francisco CA } \\
\text { (Radiology) }\end{array}$ \\
\hline Faisal Qureshi MD & fquresh@med.wayne.edu & $\begin{array}{l}\text { Hutzel Women's Hospital/Wayne } \\
\text { State University, Detroit MI } \\
\text { (Pathology) }\end{array}$ \\
\hline
\end{tabular}




\begin{tabular}{|l|l|l|}
\hline $\begin{array}{l}\text { Joseph T. Rabban III MD } \\
\text { MpH }\end{array}$ & joseph.Rabban@ucsf.edu & $\begin{array}{l}\text { University of California San } \\
\text { Francisco, San Francisco CA } \\
\text { (Pathology) }\end{array}$ \\
\hline Drucilla J Roberts,MD & $\begin{array}{l}\text { DJROBERTS@mgh.harvard. } \\
\text { edu }\end{array}$ & $\begin{array}{l}\text { Massachusetts General Hospital, } \\
\text { Harvard University, Boston MA } \\
\text { (Pathology) }\end{array}$ \\
\hline Scott Shainker DO & $\underline{\text { sshainke@bidmc.harvard.edu }}$ & $\begin{array}{l}\text { Beth Israel Deaconess Medical } \\
\text { Center, Harvard University, Boston } \\
\text { MA (Maternal \& Fetal Medicine } \\
\text { and Obstetrics \& Gynecology) }\end{array}$ \\
\hline Debra S. Heller MD & $\underline{\text { hellerds@njms.rutgers.edu }}$ & $\begin{array}{l}\text { Rutgers-New Jersey Medical } \\
\text { School, Newark NJ (Pathology) }\end{array}$ \\
\hline
\end{tabular}

Corresponding author:

Jonathan L. Hecht, MD PhD

Department of Pathology. Beth-Israel Deaconess Medical Center 330 Brookline Ave Boston, MA 02215

617-667-3817 (voice)

617-667-7120 (fax)

JLHecht@BIDMC.harvard.edu 


\begin{abstract}
.
The terminology and diagnostic criteria presently used by pathologists to report invasive placentation is inconsistent and does not reflect current knowledge of the pathogenesis of the disease or the needs of the clinical care team. A consensus panel was convened to recommend terminology and reporting elements unified across the spectrum of PAS specimens (i.e. delivered placenta, total or partial hysterectomy with or without extrauterine tissues, curetting for retained products of conception). The proposed nomenclature under the umbrella diagnosis of Placenta Accreta Spectrum (PAS) replaces the traditional categorical terminology (placenta accreta, increta, percreta) with a descriptive grading system that parallels the guidelines endorsed by the International Federation of Gynaecology and Obstetrics (FIGO). In addition, the nomenclature for hysterectomy specimens is separated from that for delivered placentas. The goal for each element in the system of nomenclature was to provide diagnostic criteria and guidelines for expected use in clinical practice.
\end{abstract}

Key Words: Placenta accreta, morbidly adherent placenta, pathology, reporting terminology 


\section{Introduction.}

Placenta Accreta Spectrum (PAS) refers to a form of abnormal placentation resulting in partial or complete retention of the placenta at the time of delivery, including placenta accreta (creta or adherenta), placenta increta, and placenta percreta. Maternal morbidity and mortality can occur from massive obstetrical hemorrhage, which often requires blood transfusion and/or hysterectomy. The rate of PAS is increasing worldwide. Observational studies from the 1970s and 1980s have reported an overall increase in the prevalence of PAS ranging from 1 in 2,510 and 1 in 4,017 compared with a rate of 1 in 533 from 1982 to 2002. In 2016 the reported incidence in the United States was 1 in 272. (1)

The leading hypothesis regarding the etiology of PAS is that defects of the endometrial-myometrial interface from uterine scarring lead to a failure of normal decidualization, which allows abnormally deep anchoring of the placental villi. Some have postulated abnormalities in the invasive properties of extravillous trophoblast, with endovascular trophoblast predominant in accreta, and interstitial extravillous trophoblast adjacent to damaged smooth muscle for increta.(2) Although PAS risk factors include advanced maternal age, multiparity, prior uterine surgeries or curettage, Asherman syndrome, and placenta previa,(3) the primary risk factor for PAS in developed countries is prior cesarean delivery. The incidence increases from $0.24 \%$ after the first cesarean delivery to $6.74 \%$ in women who have had 6 or more.(3) The screening and prenatal diagnosis of PAS is primarily made by ultrasound imaging,(4) sometimes supplemented with MRI. $(5,6)$ Accurate prenatal diagnosis is essential for planning a safe delivery in a center of excellence with a multidisciplinary team and access to adult and neonatal intensive care. (7-10) Pathology provides feedback to radiology and surgery as an important quality improvement tool and is essential to evaluate the outcome of management strategies. It is therefore crucial for pathologists to be involved in the multidisciplinary team, and to provide standardized diagnostic assessment.(11)

Intuitively, the pathologic diagnosis of PAS seems simple. The placenta is either adherent or invasive, resulting in either a delivered/evacuated placenta with an attached portion of myometrium, or a cesarean hysterectomy with placenta in-situ. Yet, a large subset of cases clinically managed as PAS are not confirmed on histology, even in the setting of a hysterectomy. This problem is illustrated by Eller et al.(12) in their study on the benefits of a multidisciplinary care team for the management of PAS. In their multicenter study, $18-29 \%$ of cases did not have pathologic confirmation of accreta despite a clinical diagnosis, although many of the pathology reports indicated thinning of the uterine wall with less than 3 mm of intervening myometrium between the placenta and the overlying serosa.(12) The distinction between abnormally adherent and invasive placentation is also problematic in the literature. A recent systematic review indicated that simple adherence (placenta accreta vera) represents about $60 \%$ of PAS, 
whereas the invasive grades, increta and percreta, represent $16 \%$ and $22 \%$ of PAS. $(13,14)$ However a large amount of heterogeneity between studies was found for all parameters. Further quantification was limited due to methodological inconsistencies with regards to clinical criteria used for the diagnosis of the condition at birth, as well as the histopathologic confirmation of the diagnosis and differential diagnosis between adherent and invasive placentation.

These clinical-pathologic discordances may affect hospital practice. For example, the diagnosis of placenta accreta in a delivered placenta may trigger more advanced imaging during a subsequent pregnancy due to the associated increased risk of PAS.(15) Reproducible grading of PAS is important in the setting of post-operative quality reviews, and allocation of resources needed for urgent management such as interventional radiology, vascular or urologic surgery.(16-18) The revised terminology will allow more detailed communication between community hospitals and specialty centers in terms of gathering data for resource allocation within the health care system.

\section{Materials and Methods.}

A consensus panel was convened within the Perinatal subcommittee of the Society for Pediatric Pathology to recommend standard terminology and reporting elements unified across the range of clinical scenarios (delivered placenta; total or partial hysterectomy with or without extrauterine tissues, and with or without an in-situ placenta; curetting for retained products of conception). Each grade of PAS was meant to identify clinically and biologically meaningful subcategories for further study.

The panel members were chosen as experts in the field and as approved representatives of societies with an interest in PAS including: the Society for Pediatric Pathology (DH,JLH, LAM, LME, PJK, RB), International Society of Gynecologic Pathology (JR), the International Federation of Placenta Associations (YK, PJK), Federation International of Gynecology and Obstetrics (EJ), International Society for Abnormally Invasive Placenta (EJ) and the Society for Maternal-Fetal Medicine (SS). The project included 5 working groups designated: Literature search, Diagnostic terminology, Basal plate with adherent myometrial fibers, Depth of invasion, Cesarean section scar dehiscence, and Future research and collaboration. Recommendations for pathology reporting were discussed at a consensus meeting of the authors in Pittsburgh on October 20, 2019.

\section{Results and Discussion.}

\section{Diagnostic terminology:}

\section{Placenta Accreta Spectrum, hysterectomy or partial myometrial resections:}

The term Placenta Accreta Spectrum (PAS) is the preferred term for the different grades of abnormal 
placental adherence and invasion in the setting of hysterectomy or partial hysterectomy. Abnormal histologic findings in delivered placentas (and curettings) are placed in a separate category from PAS, designated basal plate myometrial fibers (BPMF).

Background and context: The term PAS was chosen for consistency with recent FIGO consensus guidelines for imaging and management,(19) as approved by the Society of Gynecologic Oncology, the American College of Obstetricians and Gynecologists (ACOG), and the Society for Maternal-Fetal Medicine. $(1,20)$ Alternate terms were considered non-inclusive. In particular, 'morbidly adherent placenta,' which was used to describe simple placental retention in the $19^{\text {th }}$ century, was felt to also imply the need for a peri-partum hysterectomy, when cases may be managed expectantly or with curettage rather than hysterectomy. Likewise, the term 'abnormally invasive placenta (AIP)'(16) or 'invasive placentation'(21) suggest the requirement of invasion (i.e. increta or percreta). Other terms such as 'placental attachment disorder', or 'adhesive placenta' or 'pernicious placenta" should be avoided as they do not imply inclusion of invasive placentation. $(22,23)$

When reporting pathologic findings, the term PAS should only be applied to cases with specific macroscopic and histologic features of abnormal placentation as described below, and/or those that fulfill criteria for the clinical FIGO classification.(24) Not all cases with clinically retained placental tissue are part of the PAS. Retention can also be associated with uterine atony, chronic abruption, chorion laeve accreta, $(18,25)$ and other structural abnormalities of the uterus or placenta such as retained accessory lobes (often with an associated fibroblastic reaction), placenta previa, or entrapment or adherence of the membranes.(25)

Definition of PAS for pathologic reporting: The diagnosis of PAS after excision is based on microscopic examination of the placental bed. [Figure 1] Sections must show extended areas of absent decidua between villous tissue and myometrium. This may include areas with placental villi attached directly to the superficial myometrium or abnormal implantation with a layer of fibrinoid and intermediate trophoblast between villi and muscle. Sub-categorization of PAS, designated by Grades 1-3, is based on gross findings as discussed below. The pathologic diagnosis of PAS is restricted to hysterectomy or partial myometrial resection specimens, and cannot be made on placental tissue alone nor on biopsies of the placental bed. Abnormal histologic findings in delivered placentas (and curettings) are placed in a separate category from PAS, designated basal plate myometrial fibers (BPMF).

Gross features: The gross findings of PAS will depend on the type of specimen submitted, but are most apparent in a hysterectomy specimen with placenta in situ. Optimally, the hysterectomy is planned and dissection is guided by preoperative imaging and intraoperative evaluation. A detailed guide to specimen 
processing has been published by Dannheim et al (26) and will not be reviewed here. In areas of preserved myometrium, there are infiltrative tongues or nodules with broad pushing borders and variable thinning of the underlying uterine wall. The uterus typically shows elongation and bulging of the lower uterine segment, particularly in cases with placenta previa with uterine wall remodeling attendant upon pregnancy or due to cesarean scar dehiscence.(11) This area is often very thin and may show areas of iatrogenic surgical disruption.[Figure 2]

Dehiscence of a cesarean delivery scar with overlying placenta is interpreted by some as invasive PAS. $(11,18)$ The panel felt that these cases represent a primary uterine process rather than one of the placenta. Thus, such cases should not be categorized as invasive unless histologic features of PAS are seen in the adjacent intact placental-myometrial interface. Additional sampling will reveal areas of invasion in most cases of PAS. This issue will be discussed later (Section: Uterine scar dehiscence)

The presence of an in-situ placenta alone in a hysterectomy specimen is not diagnostic of PAS for pathologic reporting. In the absence of gross myometrial invasion, PAS should be confirmed by a gentle attempt to separate the placenta from its bed with mechanical tension to disrupt the layer of fibrinoid and blood at the maternal surface.(11, 26)[Figure 3] Sampling of the junction between the placenta and uterine wall is critical to confirm the grade of PAS and determine the lateral extent of disease. It is important to note that the adherent and invasive grades described below may coexist in the same specimen.

Microscopic features: For pathologic reporting, PAS is defined by abnormal implantation of chorionic villi upon the superficial myometrium without an intervening decidual layer. [Figure 1 and virtual slides: https://slide-atlas.org/link/opgxjx] While some cases will show chorionic villi in direct contact with myometrium, villi are frequently surrounded by fibrin and extravillous trophoblast (EVT) that form a layer overlying the myometrium. Decidual cells are distinguished by their pale eosinophilic to gray cytoplasm compared to the more amphophilic cytoplasm of EVT, and typically form sheets rather than adopt an infiltrative pattern. Immunohistochemistry for CD10 has been reported to be of value in delineating decidual cells, (26) with cytokeratin or GATA-3 stains to highlight EVT. However these are not typically necessary for diagnosis.

The formulation of a microscopic definition of PAS was controversial. There was some concern of PAS overdiagnosis in the absence of myometrial invasion, as the decidual layer may normally vary in thickness across the placental bed.(19) However, limiting the diagnosis of PAS for pathologic reporting to hysterectomy specimens ensures that the required manual examination of the placenta can be completed to confirm gross adherence. Some felt that requirement for microscopic confirmation would limit the ability 
of a pathologist to diagnose PAS in resource poor regions. The committee felt that in those situations, classification should be based on intraoperative evaluation by the surgeon using clinical criteria.(24)

The thickness of the decidual layer is variable across the placental bed, so the panel did not favor a diagnosis of accreta based on the subjective observation of a thin decidual layer, so-called "inadequate" decidualization, or in areas with retroplacental fibrin or blood clot suspicious for focal abruption.(27) Additionally, the panel felt that evidence for other features commonly seen in the setting of accreta was not sufficient for use as the sole criterion for diagnosis. Most cases of PAS will show overlapping features of tissue injury with degenerated or shriveled myofibers, interstitial edema and chronic inflammation, $(2$, $28,29)$ and remodeling (i.e. 'conversion') of outer myometrial vessels, some with intimal thickening or hemosiderin laden macrophages. [Figure 4 and virtual slides: https://slide-atlas.org/link/opzzzv and https://slide-atlas.org/link/jbwi7v ] $(26,30)$ Intrusion of intact villous tissue into massively dilated vessels at the placenta-myometrial interface is very common in all stages of PAS.[Figure 5 and virtual slide: https://slide-atlas.org/link/h7ggbf] These spaces are variably lined by endothelium or trophoblast, but at least a thin layer of media remains. $(2,31)$ An altered pattern of extravillous trophoblast (EVT) has been described in PAS. EVT is normally present in the basal plate, decidua, and inner myometrium, but deeper infiltration of the uterine wall is seen in PAS. [Figure 6 and Virtual slides: https://slideatlas.org/link/4uhfxn and cytokeratin stain: https://slide-atlas.org/link/x4bvby ]

\section{Guide to Virtual Images.}

1. Loss of the decidual layer; the defining histologic feature of PAS. Along the utero-placental interface, the decidual layer is absent at the far left of the image (compare to the right side). The contour of the interface is largely undisturbed in this example, but superficial myometrial invasion is present as loose fibrosis and chronic inflammation at the left edge of the interface. Uterine veins under the placenta are massively dilated, a finding characteristic of PAS. [https://slideatlas.org/link/opgxjx]

2. Myometrial changes deep to the placenta in PAS. In the upper section, note the massively dilated anastomosing vasculature in the mid-myometrium as well as elongation of the veins along the utero-placental interface. In the lower section, note the edema and degeneration of smooth muscle cells at the central portion of the utero-placental interface. Trophoblast conversion of subserosal vessels is also present. [https://slide-atlas.org/link/opzzzv]

3. PAS with more extensive tissue destruction. In this example, the normal low-power undulating/corrugated contour of the utero-placental interface is lost, and chronic inflammation in the myometrium with edema with myocyte injury is prominent. Organizing blood clot with entraped villi in the parenchyma of the placenta is a common finding. [https://slideatlas.org/link/jbwi7v]

4. Intrusion of intact villous tissue into a massively dilated vessel at the utero-placental interface (center-top of the image). This phenomena may give the appearance of vascular continuity 
between placenta and uterus on ultrasound, but the dilated vessels are uterine, not placental. [https://slide-atlas.org/link/h7ggbf]

5. Extravillous trophoblast extends deep into the uterine wall. This is best seen on cytokeratin stain. This example also illustrates implantation over a cesarean scar. At low power the scar appears as a pale zone across the mid-myometrium. At higher power disruption of the smooth muscle bundles, and an increase in collage and small irregular vessels are appreciated. Areas of scar are vulnerable to dehiscence (not present here). [https://slide-atlas.org/link/4uhfxn] [https://slideatlas.org/link/x4bvby (cytokeratin stain)]

6. Dehiscence of a cesarean scar (blue ink indicating the lower uterine segment abutting the bladder). In the thin band of tissue wrapping around the left edge of the placenta, notice the absence of smooth muscle. The extravillous trophoblast infiltrates to within microns of ink, and trophoblastic conversion is present in subserosal vessels. [https://slide-atlas.org/link/a4fpkr]

7. Cesarean scar dehiscence. The uterine wall is thinned and composed entirely of fibrotic scar tissue. Subserosal blood vessels are often distorted by the process, appearing dilated and tortuous. Blood clot is common in the area of scar. [https://slide-atlas.org/link/tyqijk] [https://slideatlas.org/link/tj4bee] [https://slide-atlas.org/link/sfystk (trichrome stain)]

\section{PAS grade (subcategories of PAS):}

As in the FIGO system,(24) the proposed sub-categories of PAS are designated as grades based on the degree of grossly assessed invasion and local tissue destruction.[Figure 7]

- PAS Grade 1 - non-invasive: grossly adherent placenta by manual palpation. Myometrial cross sections show a smooth placental-myometrial interface and uniform myometrial thickness without thinning.

- PAS Grade 2 - superficial invasion: cross sections show an irregular placental-myometrial interface without involvement of the outer myometrium (i.e. with preservation of at least $25 \%$ of the wall thickness relative to the uninvolved myometrium).

- PAS Grade 3A - deep invasion: cross sections show an irregular placenta-myometrial interface with involvement of the outer myometrium (i.e. with preservation of less than $25 \%$ of the wall thickness relative to the uninvolved myometrium.). The serosa is intact.

- PAS Grade 3D - deep invasion with disruption of the serosa: deeply invasive placenta with disruption of the uterine serosal surface. $(\mathrm{D}=$ deep invasion)

- PAS Grade 3E - deep invasion with adherent extrauterine structures: placental invasion into adjacent organs (most commonly bladder) or extrauterine fibroadipose tissue, confirmed by microscopy. (E= Extrauterine invasion)

Utilization: The grades loosely correspond to the AIP system (placenta accreta, increta, percreta),(16) but they are descriptive rather than categorical so that they may be harmonized with, and interpreted in the 
context of, the FIGO grades which are driven by intraoperative findings.(24) Including legacy terms (accreta, increta, percreta) alongside the above recommended terminology is discouraged, however this is left to the discretion of the local laboratory in collaboration with the clinical team.

The reason to move away from AIP categories is to improve reproducibility and decrease discrepancies from clinical diagnoses. In open discussion and case review, it became clear that the definitions of increta and percreta were variable among panel members and in the literature. For example, a recent epidemiologic review found that high heterogeneity in prevalence data between population and cohort studies is due to wide variations in diagnostic criteria.(14) Panel members all agreed on a diagnosis of placenta percreta when confirmed by histology showing chorionic villi or extravillous trophoblast in adipose tissue of the parametrium [Figure 8] or perivesical fat. However, some pathologists would accept a diagnosis of percreta in cases with chorionic villi at a disrupted, inked specimen margin without microscopic evidence of invasion into fat or based only on the surgeon's intraoperative impression. Others used the term "near percreta" in cases where sections from a thin area of uterine wall showed villi on a thin band of fibrotic tissue without residual myometrium.

The category of PAS Grade 3 deserves additional discussion. On gross examination, Grade 3A lesions show two patterns of gross morphology. The first pattern represents direct infiltrative invasion through the myometrium; on cross sections of the myometrium, these cases often show an abrupt transition from normal myometrial thickness to placental parenchyma. [Figure 9] The second pattern represents implantation adjacent to and overlying an area of cesarean scar dehiscence. Those cases show a gradual transition from myometrium to extremely thin band of fibrous tissue. [Figure 9] The area of dehiscence often comprises a large central portion of the area of placental adherence, yet may not show trophoblast infiltrating muscle and large caliber myometrial vessels. $(11,18)$ Sections from this area may even reveal decidua overlying the scar. [Figure 10] Infiltrative invasion can typically be found in sections of the placental-myometrial interface adjacent to the scar. Those sections should always be included in the pathology work-up.

Grade 3D includes disruption of the uterine wall in any form. In clinical centers without direct and immediate communication between pathologist and surgeon, it is impossible to distinguish preoperative extension beyond the uterus from surgical disruption or post-surgical manipulation of the specimen. It was felt that all specimens fragile enough to be disrupted represent a form of PAS with high risk of complication. Exceptions to this category include a highly fragmented hysterectomy, where the boundaries of the disruption and anatomic landmarks are not identified. Another example is the presence of intraoperative serosal tears unrelated to PAS, particularly if the patient has pre-existing adhesions to bladder or colon (e.g. endometriosis, prior surgeries). Extensive disruption may also occur, for example, 
if the uterus is removed by a supracervical approach to permit urgent hemostasis with subsequent removal of the cervix. Correlation with surgical and radiographic findings should guide the final diagnosis and it is acceptable to report a case as: "indefinite for intrauterine invasion, examination is limited in an area of potential surgical disruption. Correlation with surgical and radiographic findings is suggested."

Communication between pathologist and surgeon increases the accuracy of the final report (11) and may be facilitated through various means, including the specimen requisition form. Reportable elements should also include whether urinary bladder or colon wall is included en bloc with the uterus.

The pathology reporting system differs from the FIGO clinical classification for deep myometrial invasion. In the FIGO system,(24) Grade 3 PAS is divided into 3 categories based on the location of invasion: Grade 3A cases show invasion limited by the uterine serosa, Grade 3B shows urinary bladder invasion, Grade 3C shows invasion of other pelvic tissues/organs. In the resected specimen, the location of extrauterine invasion is not always clear due to disruption of the specimen. To align the pathology classification with FIGO, we removed Grades 3B and 3C from the pathology classification as both of these are captured under Grade 3E: microscopic evidence of invasion beyond the serosa. If bladder or other pelvic organs are identifiable on the specimen, they will be reported separately in the pathology template.

\section{Uterine scar dehiscence (USD)}

Definition: USD represents an incomplete disruption of the uterine wall at the site of prior cesarean delivery. USD typically occurs within the anterior lower uterine segment overlying the bladder, but may extend laterally to impinge on the parametrium. The uterine wall may become so thin that the placenta can be seen through it at delivery; a "uterine window" [Figure 16] even in the absence of abnormal placentation. $(11,18)$

Gross and microscopic features: The diagnosis of USD is based on the presence of both gross and microscopic features. On gross examination, a portion of the anterior lower uterine segment is thinned and often bulging. Bread loafed sections show a gradual wedge-shaped transition from scar to myometrium at the edge of the bulge. In contrast, infiltrative invasion shows an irregular myometrial interface without gradual transition from scar to myometrium.[Figure 9]

On histology, the uterine wall is typically only a few millimeters thick and composed entirely of fibrotic scar tissue,(32-34) sometimes with prominent branches of the uterine arteries along the serosa.[Figure 17 and virtual slides: [https://slide-atlas.org/link/a4fpkr and https://slide-atlas.org/link/tyqijk and https://slide-atlas.org/link/tj4bee and trichrome stain: https://slide-atlas.org/link/sfystk) 
When an area of USD is involved by PAS, its location and size should be documented separately from infiltrative myometrial invasion. Sections show placental villi overlying a mature, thinned scar rather than trophoblast infiltration into smooth muscle and large caliber myometrial vessels. Decidua may or may not be present along the scar. Care should be taken to distinguish uterine wall disruption (PAS Grade 3D) from invasion into adjacent tissue (PAS Grade 3E) in cases of USD, since the thin uterine wall is prone to artifactual disruption at surgery. If histologic sections do not show infiltration of trophoblast into perivesical fat or infiltrative invasion in the myometrium immediately adjacent to the defect, correlation with surgical and radiographic findings should guide the final diagnosis. It is acceptable to report a case as: "indefinite for intrauterine invasion, examination is limited in an area of potential surgical disruption. Correlation with surgical and radiographic findings is suggested.”

\section{Basal plate myometrial fibers (BPMF), fully delivered placenta and curettings samples:}

The term 'basal plate myometrial fibers' (BPMF) is the preferred term for the microscopic finding of myometrial fibers in all specimens other than hysterectomies or en-block excisions of placenta with a portion of the uterus. This will include all delivered placentas and curetting specimens.

The minimum criterion for BPMF is myometrial smooth muscle fibers attached to the basal plate of the placental disc with or without intervening decidua. A diagnosis of BPMF requires qualification including a stage and size as follows:

- $\quad$ Stage 1 - decidua present

- $\quad$ Stage 2 - decidua absent

- Size $(\mathrm{mm})$ - linear dimension along the basal plate in the largest focus

- Number of separate foci

The diagnosis of BPMF requires an explanatory note as follows: "Note: Basal plate myometrial fibers (BPMF) may be an incidental finding, but may confirm non-invasive Placenta Accreta Spectrum (PAS) in the appropriate clinical setting. Stage 1 BPMF is more likely an incidental finding than stage 2 (without intervening decidua). Clinical correlation is recommended."

Previously used synonyms: placenta accreta, occult placenta accreta, mild placenta accreta, histologic placenta accreta, basal plate myometrium, basal plate myofibers, basal plate with adherent myometrial fibers.

Background and clinical context: BPMF are identified in delivered placentas and are considered abnormal, as placentas normally separate from the uterine wall in a decidual plane (decidual separation 
zone) and should not include myometrial smooth muscle. BPMF are identified on hematoxylin and eosin (H\&E) stained placental sections of the basal plate and can be seen with variable thickness of decidua separating the muscle fibers from the basal fibrinoid/chorionic villi.(35) Diagnosis of BPMF is regarded by some as confirmation of clinical placenta accreta, (36) and their presence also portends risk for developing accreta in a subsequent pregnancy $(35,37)$, however many BPMF are seen without clinical evidence of accreta. $(35,36,38)$

The reported incidence of BPMF ranges from $0.9 \%$ to $40 \%,(35,36,38-40)$ with possible explanations for the wide range including extent of sampling or selection effects resulting from clinical indications for requesting placental examination. In the event of a macroscopically disrupted area of the basal plate (consistent with focal adherence), sampling the basal plate at the junction of intactness and disruption increases detection of BPMF, as can examination of en face sections through the basal plate.(36) BPMF have been reported to be ten times more frequent in preterm placentas than term placentas.(38) No consistent clinical risk factors associated with placenta accreta, such as advanced maternal age, multigravidity, or previous uterine instrumentation have been reported in patients with BPMF when compared with a reference group, $(36,38)$ and most do not have clinical features of placenta accreta.(35, $36,38)$

BPMF share histologic features with clinical placenta accreta [Figure 13], including decidual deficiency and increased number and thickness of extravillous trophoblast at sites of BPMF.(41) Recurrence of BPMF in subsequent pregnancies and association with PAS may be the result of focal decidual defects resulting from the delivery of a placenta with BPMF, potentially leading to inadequate decidua and abnormal trophoblast invasion in a subsequent pregnancy.(38) Placental pathologic findings associated with BPMF include decreased placental weight,(38) uteroplacental vessels with abnormal physiologic changes,(38) basal chronic villitis,(42) plasma cell deciduitis,(42) increased syncytial knots,(42) villous agglutination,(42) increased perivillous fibrin,(42) subchorionic/intervillous thrombi,(42) decidual hemosiderosis,(41) infarction,(41) and separate retroplacental blood clot.(39) Despite the association with lesions of maternal vascular malperfusion, including uteroplacental vascular abnormalities, an association between BPMF and hypertensive disorders of pregnancy was not demonstrated in the single study examining this issue.(43)

Gross features: BPMF are generally not visible grossly, with the exception being large fragments of myometrial smooth muscle adherent to the placenta. In the delivered placenta, the maternal surface may be disrupted with missing placental parenchyma, absent cotyledons, fragmentation or loss of the glistening smooth contour. [Figure 11] Directed sampling of areas of intact basal plate adjacent to the disrupted areas will increase the yield in identifying BPMF. (36) The surfaces at the junction between complete and 
incomplete parenchyma may be inked at the time of grossing for histologic correlation. These areas should be sampled perpendicular to the maternal surface in order to better visualize the relationship between muscle fibers seen along the intact maternal surface and the underlying decidua basalis. In hysterectomy after placenta delivery, obvious placental tissue is rarely apparent, so extensive sampling with attention to the cornua is needed. [Figure 12] Certainly, one can exclude invasion in these cases.

Microscopic features: BPMF are myometrial smooth muscle fibers attached to the basal plate with or without intervening decidua. The definition does not require a minimum area of involvement, but the finding should be readily apparent on routine stain such as H\&E. Screening using immunohistochemistry is not recommended at this time.

Myometrial fibers attached to the basal plate of the placenta along the plane of separation are easily recognized on H\&E-stained sections of the basal plate oriented on edge, but must be differentiated from decidua and trophoblast. When the myometrial fibers are hypertrophic, distinguishing features include smooth muscle present as small bundles or clusters oriented either longitudinally or on cross section. The hypertrophic BPMF can demonstrate disarray.(39) BPMF that are shriveled, degenerated, [Figure 13D] or frankly necrotic have been described in association with retroplacental blood, and these may require actin staining for verification.(39) Smooth muscle that might be a portion of venous wall should be differentiated from BPMF. In the most superficial cases of BPMF, myometrial fibers are separated from the basal fibrinoid/chorionic villi by a relatively thick layer of decidua.(35) Less frequently, the decidual layer between chorionic villi and myometrium is reduced or is entirely absent.(35) Sections taken en face adjacent to areas of disruption have been shown to increase detection of BPMF,(36) however, these sections do not allow determination of the amount of decidua present.

There are some cases in which the presence of myometrium may not be straightforward. Smooth muscle around decidual vessels can be mistaken for but differentiated from myometrium by their thin fibers that run parallel to the adjacent vessel. Myometrium tends to have myofibers cut both longitudinally and transversely.

Immunohistochemical staining: Immunohistochemical staining for actin highlights myometrial smooth muscle, but is not indicated when myometrial smooth muscle is readily apparent on H\&E stained sections. In a study of BPMF in association with retroplacental hemorrhage, Wyand et al employed an evidencebased approach to investigate when actin staining is indicated in the presence of retroplacental hemorrhage, and suggested the following indications: 1) when BPMF are suspected on routine H\&E stains but need confirmation; 2) when BPMF are not suspected on routine H\&E slides, but the history suggests possible accreta (e.g. prior cesarean delivery, placenta previa, manual removal, retained placenta, gross 
disruption of the maternal surface, prior history of accreta, or ultrasound suspicion of accreta); 3) when dilated endometrial gland remnants and infiltrative chorion are identified in sections of fetal membranes, a finding associated with morbidly adherent fetal membranes in association with retained placenta; 4) after identification of decidual hemosiderosis, a finding associated with prior retroplacental bleeding.(39) Although immunostains do increase the sensitivity for detecting BPMF, the specificity of BPMF that are not readily apparent on H\&E is lower with respect to clinically significant disease.(27).

Immunohistochemistry for BPMF is certainly useful as a teaching adjunct.

Curettings and fragmented samples: Specimens from evacuation procedures most often come from patients with post-partum hemorrhage who are found to have retained products of conception on ultrasound. The diagnosis of BPMF can rarely be made in retained placental fragments and post-delivery endometrial curettings, but care must be taken to exclude artifacts of tissue retention and specimen fragmentation. For example, retained placental tissue may show areas of perivillous fibrin that obscure the decidua; subinvolution of the implantation site may include incomplete trophoblastic transformation of large myometrial vessels and fibrin thrombi as well as acute and chronic inflammation that mimic invasive villous tissue. Tissue may be also damaged following interventional radiology or the use of methotrexate which may eventually lead to the spontaneous delivery of the placenta weeks or months after the birth. These artifacts are illustrated in [Figure 14]

Stage and size: Because a variable amount of basal decidua can separate the BPMF from the basal fibrinoid/chorionic villi, it has been suggested that the spectrum of BPMF be defined by the amount of decidua between the myometrium and basal fibrinoid/chorionic villi.(35) In the staging system developed by Linn et al, which stages PAS as stage 0 (normal) to stage 6 (placenta percreta with invasion of or attachment to adjacent organs), BPMF are included in stages 1 through stage 3 . Specifically, stage 1 is BPMF attached at the separation zone of the basal plate with numerous layers of intervening decidua present; stage 2 is BPMF with the decidual layer between chorionic villi and myometrium reduced to 2 cell layers or less; and stage 3 is BPMF is contact with basal fibrinoid/chorionic villi without intervening decidua. [Figure 15]

Linn et al also developed a method of quantification of BPMF utilizing the total length of the basal plate examined microscopically, the largest focus of BPMF, and total length of BPMF measured using an ocular micrometer. The percentage of BPMF was then calculated by dividing the total length of BPMF by the total length of basal plate examined microscopically. Furthermore, they performed a validation study demonstrating that their diagnostic sampling was proportionate to the amount of BPMF along the entire basal plate. (35) 
Utilizing this staging and quantification, Linn et al demonstrated that BPMF stage 2 or 3 is a risk factor for placenta accreta in a subsequent pregnancy.(35) They also found that a greater quantity of BPMF $(>1.5 \%)$ of the sampled basal plate is a risk factor for placenta accreta regardless of stage. The finding of BPMF stage 1 or smaller quantity of BPMF did not correlate with subsequent development of accreta in the next pregnancy, suggesting a threshold amount must be reached in order to increase the risk. Therefore they proposed that stage 2 BPMF can be called "BPMF with changes suggestive of focal accreta", while stage 3 can be called "histologic accreta". (35)

The panel felt that the evidence for the clinical importance of quantity has not been sufficiently validated. Prospective controlled studies and additional data from studies of clinical correlation with quantity of adherent myometrium will be needed before it is included in the BPMF explanatory note. For now, the panel suggests including the linear dimension along the basal plate in the largest focus of BPMF, measured using an ocular micrometer or measured on a scanned slide or digital image. [Figure 13A] Utilization: The proposed pathologic definition for PAS is for hysterectomy specimens. In addition, focal areas of placental invasion can be removed by partial myometrial resection to avoid a peri-partum hysterectomy and provide the pathologist with a sample containing both invasive villous tissue and myometrium suitable for grading PAS.

Separating the terminology for delivered placentas and hysterectomy specimens was one of the more controversial issues discussed. We recognize that some cases of delivered placentas requiring instrumentation or complicated by maternal hemorrhage represent PAS, but the specificity of BPMF for clinically significant disease or invasive grades of PAS was low relative to clinical variables. Unlike the FIGO classification,(24) we do include delivered placentas in the overall system of terminology under the heading of Basal Plate Myometrial Fibers. The accompanying explanatory note was developed to link BPMF to the PAS system by acknowledging that a subset of cases are physiologically similar to PASGrade 1.

More clinical data is needed to develop management recommendations for women with asymptomatic BPMF. (39) Although the presence of BPMF is associated with PAS in future pregnancies, the specificity of BPMF as a predictor of morbidity is low when applied as an isolated finding. BPMF is common, with an incidence in reported series of $8 \%$ to $30 \%$ with extended sampling. $(36,38)$ Looking at paired samples (index and prior pregnancy), Miller et al. found BPMF in the previous placenta in $40 \%$ of controls (those without BPMF or PAS in the index pregnancy).(37) The finding of BPMF was clinically occult in $31 \%$ of cases reported by Heller.(27) In addition, although BPMF in an index pregnancy are associated with BPMF in a subsequent pregnancy, the risk factor for PAS with invasion is unclear. Therefore, as stated in 
the required explanatory note, a diagnosis of BPMF should be interpreted in the context of other clinical predictors of PAS, such as placenta previa, history of cesarean section, and abnormal imaging. We do not make any specific management recommendations as all published series have been retrospective with no prospective trials of increased surveillance based on BPMF.

\section{Reporting format}

Pathology reports should include a general categorization of PAS with a grade. A gross description should specify the anatomic location of implantation and an estimate of the area of the uterine wall involved. This area can be enumerated in $\mathrm{cm}^{2}$ or a percentage of the specified area (e.g. placental invasion involving $20 \%$ of the placental bed in the lower uterine segment extending into to the lower fundus, and right lateral wall; placental invasion over $100 \%$ of the placental bed with a $2 \times 2 \mathrm{~cm}$ disruption of the right lower uterine segment and histologic extension of non-villous trophoblast into parametrial fat).

Methods to assess and categorize the extent of involvement of the placental bed have been published.(44) In that system, "focal placenta accreta" involves only one lobule, either partially or entirely; "total placenta accreta" involves of all placental lobules, or "partial placenta accreta" involves at least two, but not all placental lobules. The panel felt that the terms "focal", "total" and "partial" did not convey sufficient anatomic detail for correlation with clinical and imaging findings. In addition, evaluation for extent of adherence in manually removed placentas is limited since those affected by PAS are frequently distorted by attempts at manual removal.(11, 18, 34, 45) For now, the report should include the location and extent of involvement as an estimated percentage of the placental bed.

Quantification of the depth of invasion deserves further study. Any scoring system for depth of invasion will need to separate the effects of placental invasion from uterine wall remodeling as seen in cesarean scar dehiscence. If histologic features of PAS are not present, a statement suggesting clinical correlation should be included.

The panel decided to include 'basal plate myometrial fibers' separately from PAS, and with a descriptive note. BPMF are often detected incidentally on delivered placentas based on random sectioning (with no associated gross findings), so quantitation based on gross features is difficult. This lesion should be qualified by stage, the number of foci, and linear measurement of the largest focus.

\section{-BOX------------}

Report template:

Specimens: uterus post vaginal delivery, uterus post cesarean delivery, uterus with placenta in-situ, placenta via vaginal delivery, placenta via cesarean delivery, curettings 
I. Placenta accreta spectrum (PAS),

- PAS Grade [1-3]

- Other reportable elements (as applicable):

1. Location of implantation in the uterus

2. Estimated \% of the disc that is adherent to the myometrium or invasive

3. Location of invasive component

1. Lower uterine segment (adjacent to bladder)

2. Anterior wall (above bladder)

3. Posterior wall

4. Lateral wall / broad ligament.

4. Maximum $\%$ invasion $<25 \%, 25-50 \%, 51-75 \%,>75 \%$

5. Location of serosal disruption

1. Area $\left(\mathrm{cm}^{2}\right)$ of serosal disruption

2. Presence of adherent organs (bladder, colon)

6. Implantation over cesarean scar dehiscence

II. Basal plate myometrial fibers (BPMF)

1. Stage $[1-2]$

2. Size $(\mathrm{mm})$ - linear dimension along the basal plate in the largest focus

3. Number of separate foci

Note: Basal plate myometrial fibers (BPMF) may be an incidental finding, but may confirm non-invasive Placenta Accreta Spectrum (PAS) in the appropriate clinical setting. Stage 1 BPMF is more likely an incidental finding than stage 2 (without intervening decidua). Clinical correlation is recommended.

BOX--------

\section{New directions.}

Several areas of further investigation were proposed during the meeting. 
1. The incidence of BPMF in uncomplicated pregnancies.

2. Additional studies on the clinical significance and risk factors of BPMF.

3. Changes in the reported incidence of PAS and BPMF among cases classified using the proposed classification relative to original reported diagnosis.

4. Clinical complications and outcome for each PAS grade.

\section{Acknowledgements:}

We acknowledge the Society for Pediatric Pathology leadership and UPMC Children's Hospital Pathology (Sara Vargas and Miguel Reyes-Múgica MD) for hosting the consensus conference. Kate Dannheim, MD for acting as meeting secretary and draft editor. Kate is an Assistant Professor of Pathology, Warren Alpert Medical School of Brown University, Surgical and Pediatric Pathologist at Rhode Island and Hasbro Children's Hospitals. Beverly Faulkner-Jones, MD for assistance with SlideAtlas, our whole slide imaging platform (http://slideatlas.kitware.com/)

\section{Conflicting Interests statement:}

The Authors declare that there is no conflict of interest. 


\section{References:}

1. Obstetric Care Consensus No. 7: Placenta Accreta Spectrum. Obstet Gynecol 2018;132:e259-e75.

2. Cramer SF, Heller DS. Placenta Accreta and Placenta Increta: An Approach to Pathogenesis Based on the Trophoblastic Differentiation Pathway. Pediatr Dev Pathol 2016;19:320-33.

3. Silver RM, Landon MB, Rouse DJ, Leveno KJ, Spong CY, Thom EA, et al. Maternal morbidity associated with multiple repeat cesarean deliveries. Obstet Gynecol 2006;107:1226-32.

4. Jauniaux E, Ayres-de-Campos D, Diagnosis FPA, Management Expert Consensus P. FIGO consensus guidelines on placenta accreta spectrum disorders: Introduction. Int J Gynaecol Obstet 2018;140:261-4.

5. Berkley EM, Abuhamad AZ. Prenatal diagnosis of placenta accreta: is sonography all we need? J Ultrasound Med 2013;32:1345-50.

6. Reddy UM, Abuhamad AZ, Levine D, Saade GR. Fetal Imaging Workshop Invited P. Fetal imaging: executive summary of a joint Eunice Kennedy Shriver National Institute of Child Health and Human Development, Society for Maternal-Fetal Medicine, American Institute of Ultrasound in Medicine, American College of Obstetricians and Gynecologists, American College of Radiology, Society for Pediatric Radiology, and Society of Radiologists in Ultrasound Fetal Imaging Workshop. J Ultrasound Med 2014;33:745-57.

7. Committee opinion no. 529: placenta accreta. Obstet Gynecol 2012;120:207-11.

8. Bailit JL, Grobman WA, Rice MM, Reddy UM, Wapner RJ, Varner MW, et al. Morbidly adherent placenta treatments and outcomes. Obstet Gynecol 2015;125:683-9.

9. Silver RM, Fox KA, Barton JR, Abuhamad AZ, Simhan H, Huls CK, et al. Center of excellence for placenta accreta. Am J Obstet Gynecol 2015;212:561-8.

10. Shamshirsaz AA, Fox KA, Salmanian B, Diaz-Arrastia CR, Lee W, Baker BW, et al. Maternal morbidity in patients with morbidly adherent placenta treated with and without a standardized multidisciplinary approach. Am J Obstet Gynecol 2015;212:218 e1-9.

11. Jauniaux E, Hussein AM, Zosmer N, Elbarmelgy RM, Elbarmelgy RA, Shaikh H, et al. A new methodologic approach for clinico-pathologic correlations in invasive placenta previa accreta. Am J Obstet Gynecol 2019.

12. Eller AG, Bennett MA, Sharshiner M, Masheter C, Soisson AP, Dodson M, et al. Maternal morbidity in cases of placenta accreta managed by a multidisciplinary care team compared with standard obstetric care. Obstet Gynecol 2011;117:331-7.

13. Abuhamad A. Morbidly adherent placenta. Semin Perinatol 2013;37:359-64.

14. Jauniaux E, Bunce C, Gronbeck L, Langhoff-Roos J. Prevalence and main outcomes of placenta accreta spectrum: a systematic review and metaanalysis. Am J Obstet Gynecol 2019. 
15. Mullen C, Battarbee AN, Ernst LM, Peaceman AM. Occult Placenta Accreta: Risk Factors, Adverse Obstetrical Outcomes, and Recurrence in Subsequent Pregnancies. Am J Perinatol 2018.

16. Collins SL, Ashcroft A, Braun T, Calda P, Langhoff-Roos J, Morel O, et al. Proposal for standardized ultrasound descriptors of abnormally invasive placenta (AIP). Ultrasound Obstet Gynecol 2016;47:271-5.

17. Jauniaux E, Bhide A, Kennedy A, Woodward P, Hubinont C, Collins S, et al. FIGO consensus guidelines on placenta accreta spectrum disorders: Prenatal diagnosis and screening. Int J Gynaecol Obstet 2018;140:274-80.

18. Jauniaux E, Hussein AM, Fox KA, Collins SL. New evidence-based diagnostic and management strategies for placenta accreta spectrum disorders. Best Pract Res Clin Obstet Gynaecol 2019;61:75-88.

19. Jauniaux E, Collins S, Burton GJ. Placenta accreta spectrum: pathophysiology and evidence-based anatomy for prenatal ultrasound imaging. Am J Obstet Gynecol 2018;218:75-87.

20. Society of Gynecologic O, American College of O, Gynecologists, the Society for Maternal-Fetal M, Cahill AG, Beigi R, et al. Placenta Accreta Spectrum. Am J Obstet Gynecol 2018;219:B2-B16.

21. Steins Bisschop CN, Schaap TP, Vogelvang TE, Scholten PC. Invasive placentation and uterus preserving treatment modalities: a systematic review. Arch Gynecol Obstet 2011;284:491-502.

22. Collins SL, Chantraine F, Morgan TK, Jauniaux E. Abnormally adherent and invasive placenta: a spectrum disorder in need of a name. Ultrasound Obstet Gynecol 2018;51:165-6.

23. Jauniaux E, Collins SL, Jurkovic D, Burton GJ. Accreta placentation: a systematic review of prenatal ultrasound imaging and grading of villous invasiveness. Am J Obstet Gynecol 2016;215:712-21.

24. Jauniaux E, Ayres-de-Campos D, Langhoff-Roos J, Fox KA, Collins S, Diagnosis FPA, et al. FIGO classification for the clinical diagnosis of placenta accreta spectrum disorders. Int J Gynaecol Obstet 2019;146:20-4.

25. Khong TY, Cramer SF, Heller DS. Chorion laeve accreta - Another manifestation of morbid adherence. Placenta 2018;74:32-5.

26. Dannheim K, Shainker SA, Hecht JL. Hysterectomy for placenta accreta; methods for gross and microscopic pathology examination. Arch Gynecol Obstet 2016;293:951-8.

27. Heller DS, Wyand R, Cramer S. Recurrence of Basal Plate Myofibers, with Further Consideration of Pathogenesis. Fetal and pediatric pathology 2019;38:30-43.

28. Ernst LM, Linn RL, Minturn L, Miller ES. Placental Pathologic Associations with Morbidly Adherent Placenta: Potential insights into Pathogenesis. Pediatr Dev Pathol 2016.

29. Khong TY, Robertson WB. Placenta creta and placenta praevia creta. Placenta 1987;8:399-409. 
30. Tantbirojn P, Crum CP, Parast MM. Pathophysiology of placenta creta: the role of decidua and extravillous trophoblast. Placenta 2008;29:639-45.

31. Parra-Herran C, Djordjevic B. Histopathology of Placenta Creta: Chorionic Villi Intrusion into Myometrial Vascular Spaces and Extravillous Trophoblast Proliferation are Frequent and Specific Findings With Implications for Diagnosis and Pathogenesis. Int J Gynecol Pathol 2016;35:497508.

32. Timor-Tritsch IE, Monteagudo A, Cali G, Palacios-Jaraquemada JM, Maymon R, Arslan AA, et al. Cesarean scar pregnancy and early placenta accreta share common histology. Ultrasound Obstet Gynecol 2014;43:383-95.

33. van der Voet LF, Bij de Vaate AM, Veersema S, Brolmann HA, Huirne JA. Long-term complications of caesarean section. The niche in the scar: a prospective cohort study on niche prevalence and its relation to abnormal uterine bleeding. BJOG 2014;121:236-44.

34. Fox H. PLACENTA ACCRETA, 1945-1969. Obstetrical \& Gynecological Survey 1972; 27:47590.

35. Linn RL, Miller ES, Lim G, Ernst LM. Adherent basal plate myometrial fibers in the delivered placenta as a risk factor for development of subsequent placenta accreta. Placenta 2015;36:141924.

36. Khong TY, Werger AC. Myometrial fibers in the placental basal plate can confirm but do not necessarily indicate clinical placenta accreta. Am J Clin Pathol 2001;116:703-8.

37. Miller ES, Linn RL, Ernst LM. Does the presence of placental basal plate myometrial fibres increase the risk of subsequent morbidly adherent placenta: a case-control study. BJOG 2016;123:2140-5.

38. Sherer DM, Salafia CM, Minior VK, Sanders M, Ernst L, Vintzileos AM. Placental basal plate myometrial fibers: clinical correlations of abnormally deep trophoblast invasion. Obstet Gynecol 1996;87:444-9.

39. Wyand R, Cramer SF, Oshri A, Heller DS. Association of Retroplacental Blood With Basal Plate Myofibers. Pediatr Dev Pathol 2018;21:371-9.

40. Jacques SM, Qureshi F, Trent VS, Ramirez NC. Placenta accreta: mild cases diagnosed by placental examination. Int J Gynecol Pathol 1996;15:28-33.

41. Stanek J, Drummond Z. Occult placenta accreta: the missing link in the diagnosis of abnormal placentation. Pediatr Dev Pathol 2007;10:266-73.

42. Ernst LM, Linn RL, Minturn L, Miller ES. Placental Pathologic Associations With Morbidly Adherent Placenta: Potential Insights Into Pathogenesis. Pediatr Dev Pathol 2017;20:387-93.

43. Wang AA, Ernst LM, Miller ES. Basal Plate Myometrial Fibers and Hypertensive Disorders of Pregnancy: A Case-Control Study. Pediatr Dev Pathol 2018;21:296-9. 
44. Wortman AC, Alexander JM. Placenta accreta, increta, and percreta. Obstet Gynecol Clin North Am 2013;40:137-54.

45. Timor-Tritsch IE, Monteagudo A, Cali G, Vintzileos A, Viscarello R, Al-Khan A, et al. Cesarean scar pregnancy is a precursor of morbidly adherent placenta. Ultrasound Obstet Gynecol 2014;44:346-53. 


\section{Figures:}

Figure 1. Normal implantation and PAS. PAS is defined as the loss of decidual layer between villi and myometrium (inset). H\&E stained section at 100x original magnification.

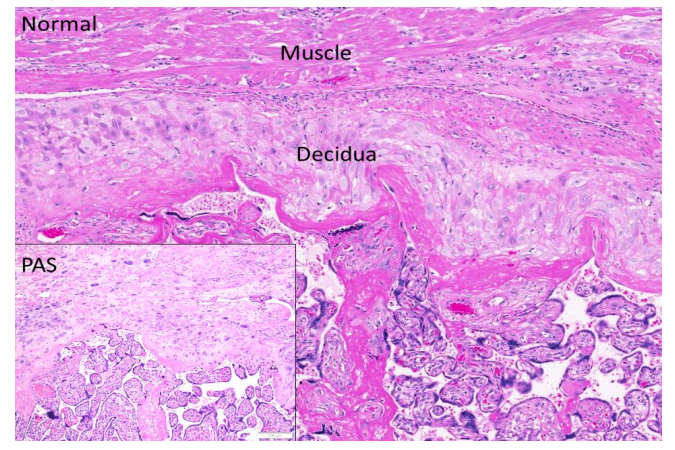

Figure 2. Uterine remodeling. Distension and bulging of the lower uterine segment with prominent serosal vasculature in a case of placenta previa $\left(\mathrm{A},{ }^{*}\right)$. This remodeling is often associated with dehiscence of a cesarean section scar. B. Thin areas of the uterine wall are prone to disruption either due to invasion beyond the serosa or to manipulation of the specimen during and after surgery. The incision used for delivery is indicated by an arrow.

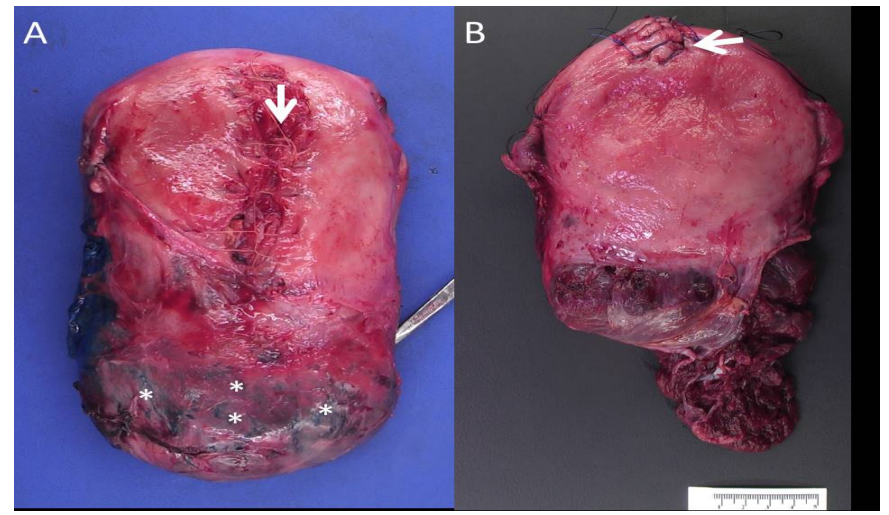

Figure 3. A. Cross section of normal placenta attached to the uterine wall. On the left, a portion of the placenta remained attached after gentle mechanical tension. There is a thick undulating layer of fibrinoid and dilated vessels along the interface (arrow). On the right, an uninvolved area where the disc is largely separated from the myometrium and the roof and base of the gap are smooth contoured. B-C. Low and high power microscopic images of sections from the left-hand image in panel A. D. Cytokeratin stain demonstrates sheets of trophoblast in fibrinoid along the utero-placental interface.

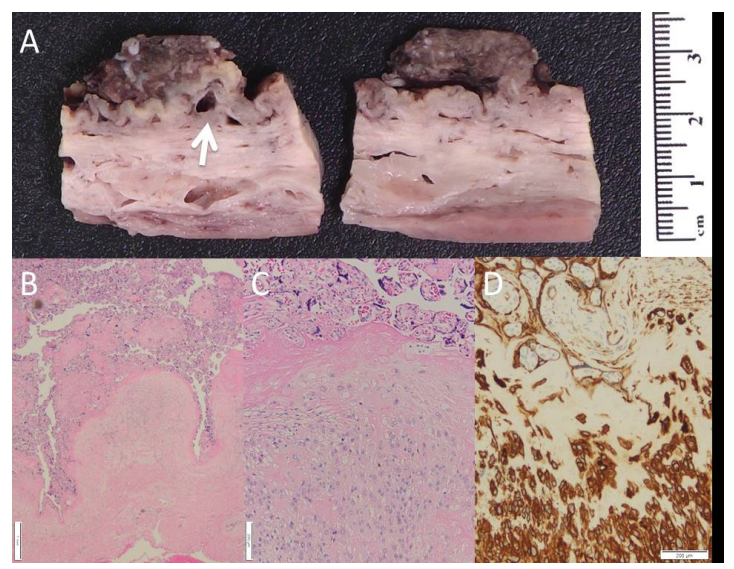


Figure 4. A. A case of PAS showing infiltrative invasion with fibrosis replacing myofibers accompanied by increased chronic inflammation (left) with edema (right). B-C. Deeper sections show trophoblastic remodeling of outer myometrial vessels (arrows).

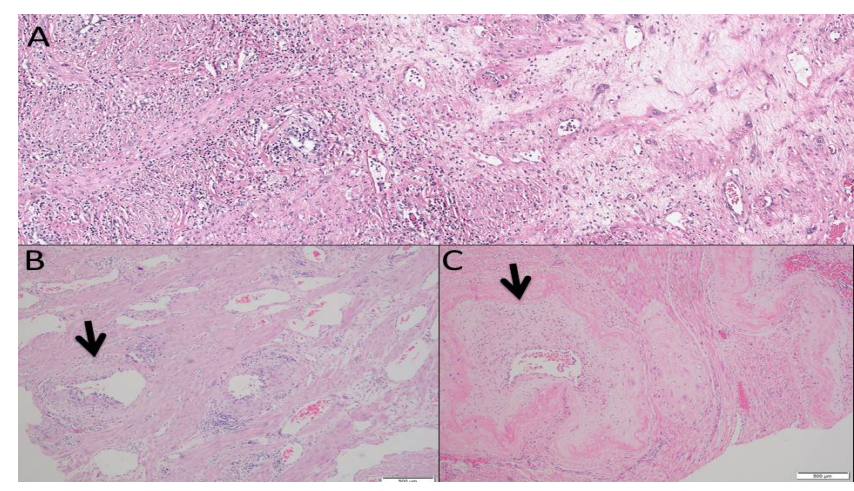

Figure 5. Villous tissue in dilated myometrial vessels at the invasive front (arrow). This, along with remodeled "converted" arteries may give the appearance of vascular continuity between placenta and uterus, but the dilated vessels are uterine, not placental.

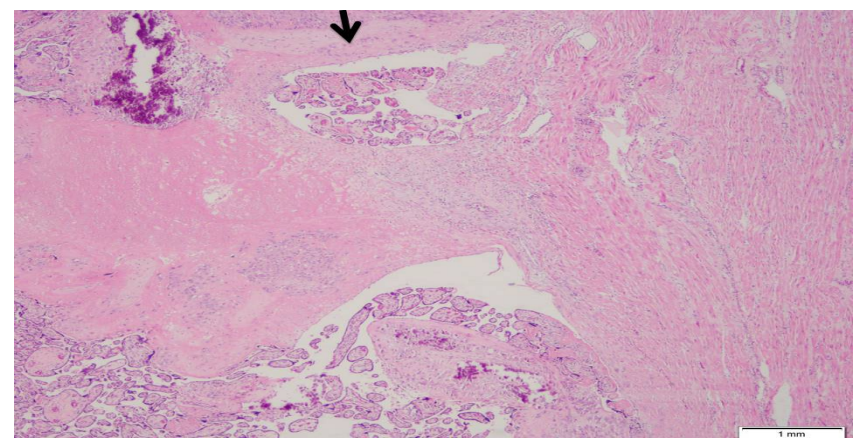

Figure 6. A case of PAS demonstrating an altered pattern of extravillous trophoblast, extending well beyond the decidua and inner myometrium, best seen on cytokeratin stain. H\&E and cytokeratin stain at scanning magnification; chorionic villi are present at the right of each image. This example also illustrates implantation over a cesarean scar. At low power the scar appears as a pale zone across the midmyometrium (arrow). At higher power (https://slide-atlas.org/link/4uhfxn) disruption of the smooth muscle bundles, and an increase in collage and small irregular vessels are appreciated.

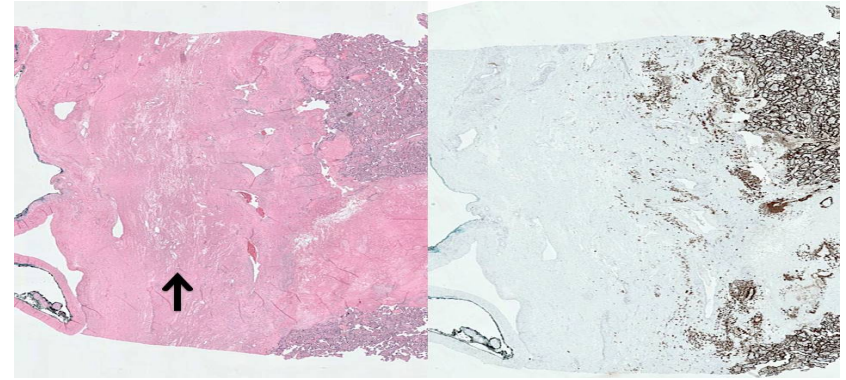

Figure 7. A. Schematic of the PAS grading system. A layer of decidualized endometrium normally separates the placenta from the myometrium. The diagnostic feature of PAS is the loss of this decidual layer. PAS is graded based on the degree of disruption of the uterine wall. Grade 1 is non-invasive without gross thinning of the uterine wall. Grade 2 shows thinning of the uterine wall below the placenta, with preservation of at least $25 \%$ of the wall thickness relative to uninvolved myometrium. Grade $3 \mathrm{~A}$ shows thinning of the uterine wall below the placenta, with preservation of less than $25 \%$ of the wall thickness 
relative to uninvolved myometrium. Grade 3D shows disruption of the uterine serosa and Grade 3E shows invasion into extrauterine structures. B. PAS Grades 2-3 often occur in the setting of a cesarean scar dehiscence. The placenta may be adherent to the scar (arrow), but a pathologic diagnosis of PAS requires sections showing myometrial invasion. Thus sectioning in areas away from the scar is essential.

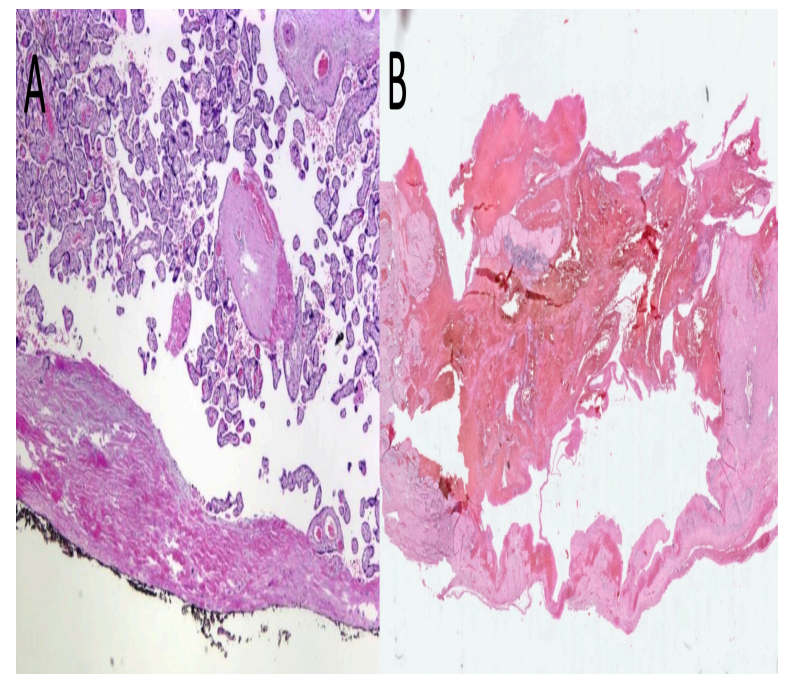

Figure 8. PAS Grade 3E. The bladder is adherent to the uterine serosa with fibrosis obscuring the outer limit of the uterus (arrows indicate the boundary of the uterus). A pale area beyond the uterine serosal boundary $(*)$ represents trophoblast infiltration at high power. $\mathrm{BM}=\mathrm{Bladder}$ mucosa, $\mathrm{P}=\mathrm{Placenta}$.

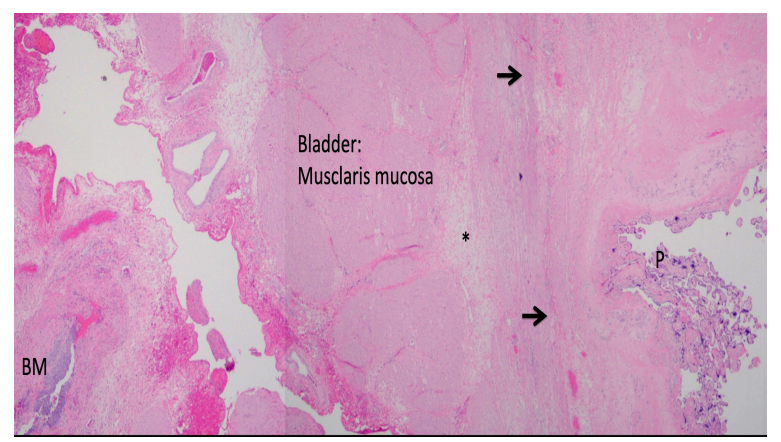

In the new terminology, Grade 3E only requires the presence of EVT within tissues outside the uterus, rather than villi extending through the bladder wall into the lumen. Unlike the clinical FIGO classification, Grade 3 in the pathologic classification system does not specify the location of extrauterine invasion. However, this case represents invasion adjacent to the bladder and could also be classified clinically as FIGO Grade 3B.

Figure 9. Patterns of invasion. Top slice: Tongues of invasion represent cancer-like infiltration of myometrium (*). Bottom Slice: Broad pushing invasion represents a component of cesarean scar dehiscence (-). Classifying cases with placenta adherent to a scar are problematic, and the grade should not be assigned based on the area of scar dehiscence alone. Sections should include adjacent myometrium which typically show diagnostic features of PAS. Adjacent sections show a wedge shaped transition from thick myometrium to thin scar (marked by a +, also see Figure 10) and would warrant a Grade 3A.

Typically cases of PAS also show a mixed pattern of infiltration, and PAS can be diagnoses and graded on areas away from the scar (upper slice). 


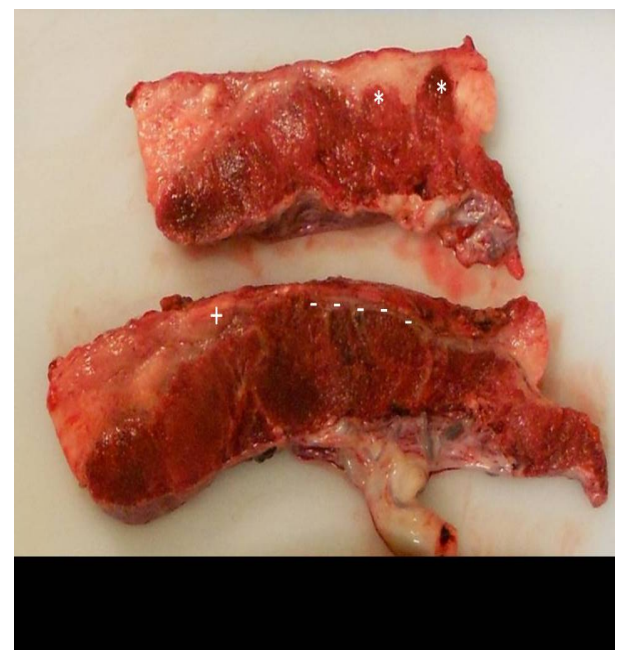

Figure 10: Figure 10: Transition from myometrium to cesarean scar dehiscence. An area of scar dehiscence is often present in the center of the implantation site of PAS. As seen grossly in figure 9, there is a triangular wedge-shape transition $(+)$ from myometrium to a thin layer of fibrous tissue in the region of scar dehiscence. Classifying cases as PAS based on sections of the scar may not be possible. In some cases, residual decidua may be present $(*$ in high magnification insert) or disrupted by blood clot (see figure 17).

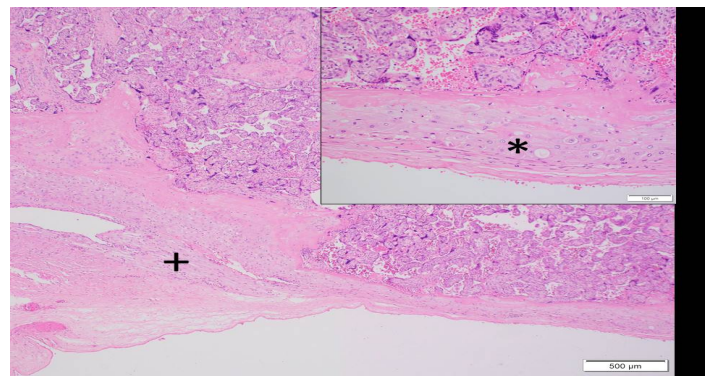

Figure 11. A delivered placenta with maternal surface disruption. Sampling should be directed at the edge of areas where the maternal surface is disrupted (arrow). Sections should be perpendicular, but do not need to include the full disc thickness, so that multiple sections can be submitted in one cassette.

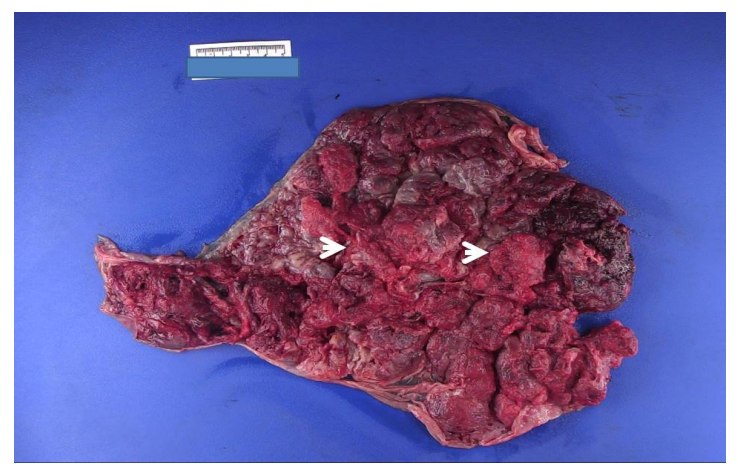

Figure 12. Post delivery hysterectomy for hemorrhage. Areas with adherent placenta may be difficult to identify in the uterine cavity in the setting of delivered placenta. The surface is dominated by blood clot and decidua. 


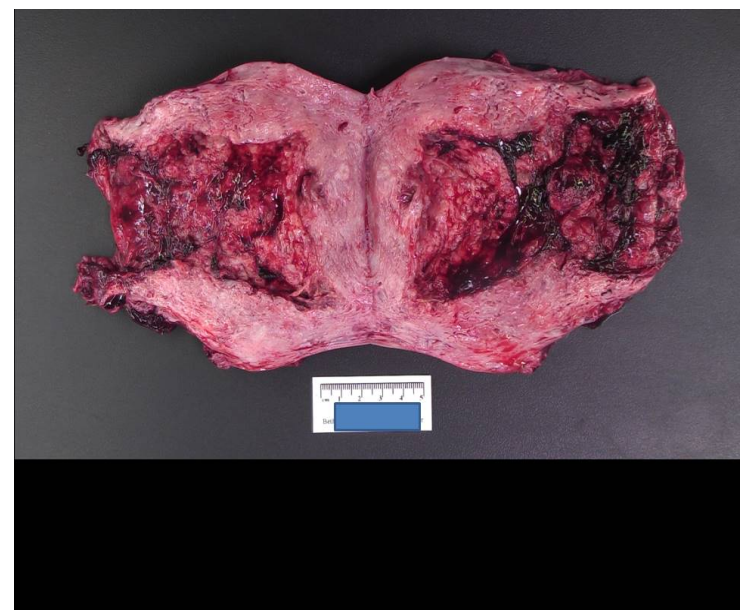

Figure 13. Basal plate with adherent myometrial fibers. The number of foci and size of the largest focus should be measured along the maternal surface (A). A stage should be assigned based on the presence or absence of decidua. In B, a layer of decidual cells (arrow) and extravillous trophoblast cells are still present between the chorionic villi and the adherent myometrial fibers (stage 1). In $\mathrm{C}$, that layer is lost (stage 2). Panel D illustrates BPMF that are shriveled and degenerated. These may require actin staining for verification.

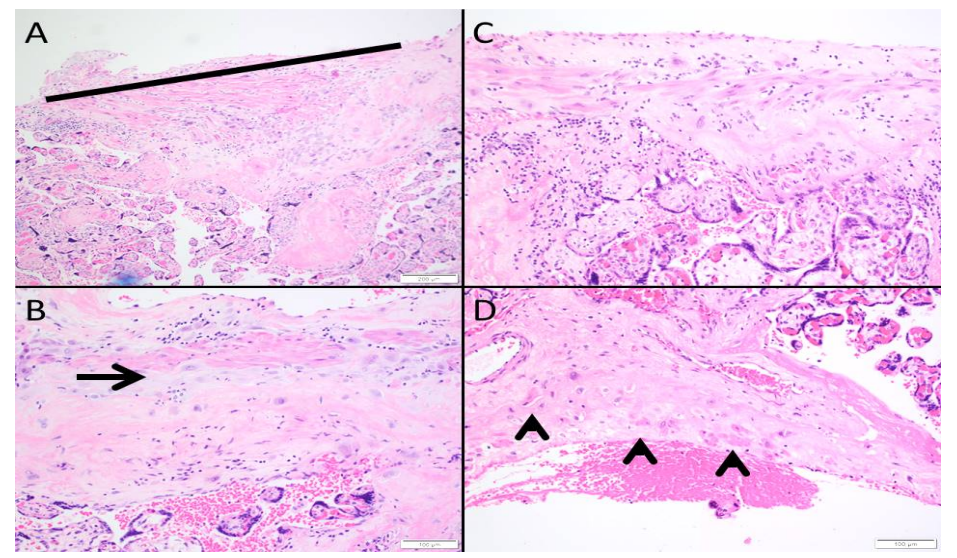

Figure 14. Indeterminate for BPMF in a fragmented sample. Uterine curettings in the setting of retained products of conception may juxtapose myometrial smooth muscle with degenerated villous tissue (arrow). It is often impossible to determine whether this is placental adherence or artifact.

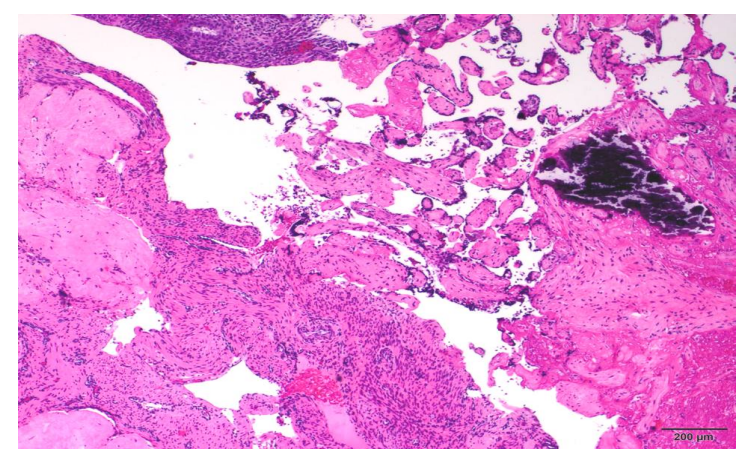

Figure 15. Sections from a delivered placenta showing BPMF, taken perpendicular to the maternal surface. Only EVT with fibrinoid separate villi from a band of smooth muscle. 


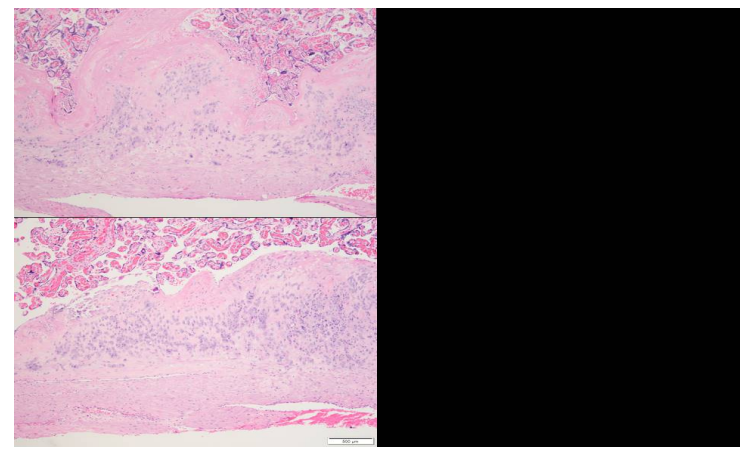

Figure 16. A myometrial window (*) within a thinned lower uterine segment (LUS). The finding likely represents dehiscence of a cesarean delivery scar. This is not sufficient evidence of PAS as only membranes are present over this thinned area. The delivery incision at the fundus is indicated by arrows.

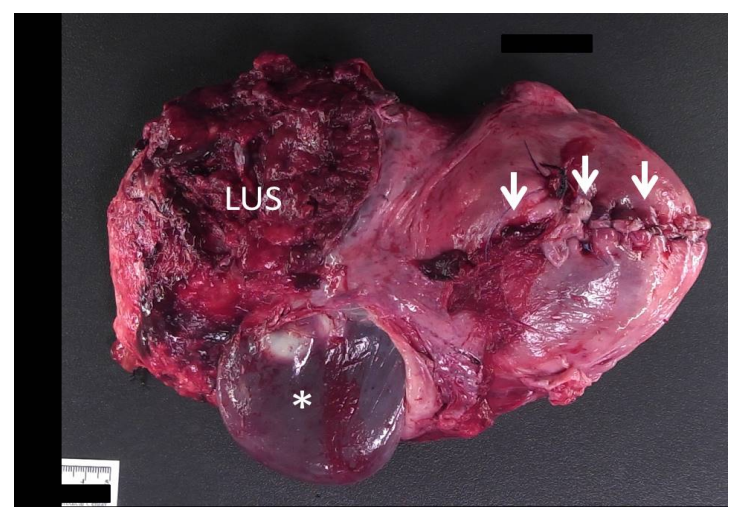

Figure 17. Cesarean scar dehiscence. In cases of cesarean scar dehiscence, the anterior lower uterine segment has a thin area of fibrosis. On histology, the uterine wall is only a few millimeters thick and composed entirely of fibrotic scar tissue. Sections may show villi adherent to scar (A) or just blood and fibrin (B), but neither finding meets criteria for PAS.

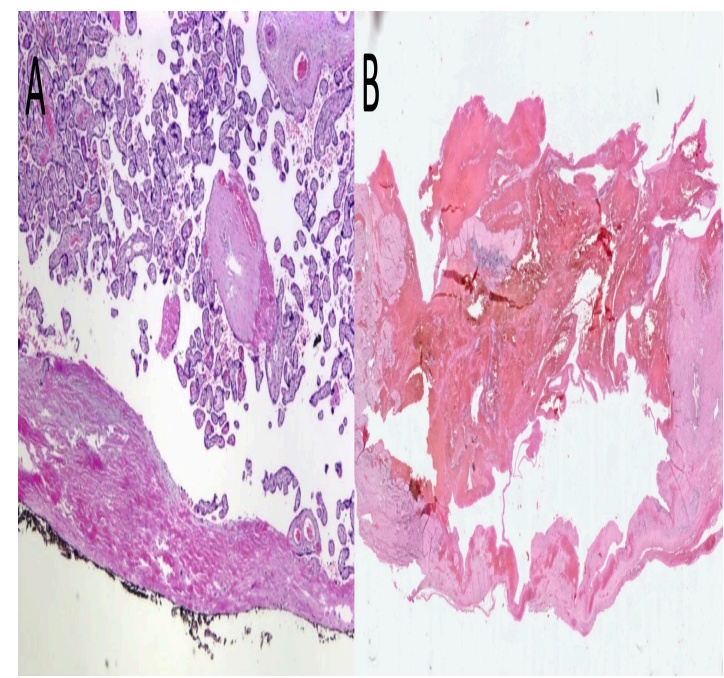

